\title{
Doença inflamatória intestinal no idoso
}

Marta Brenner Machado

Luciana Guedes

\section{INTRODUÇÃO}

A incidência e a prevalência da doença inflamatória intestinal (DII) vêm apresentando uma curva ascendente e, considerando a população idosa, os números podem aumentar mais no futuro. A apresentação clínica e a história natural da doença diagnosticada no idoso têm muitas semelhanças com aquelas exibidas na população jovem, mas importantes diferenças devem ser motivo de especial atenção, como é o caso do diagnóstico diferencial. Dados específicos da "maior idade" com DII são escassos, até porque geralmente este é um grupo excluído dos grandes ensaios clínicos, o que torna o manejo e a tomada de decisão algo bastante desafiador. A escolha do tratamento no paciente idoso com DII precisa levar em conta os comemorativos próprios desta faixa etária, que são as comorbidades, as funções locomotora e cognitiva, a polifarmácia, as queixas muitas vezes imprecisas, as ansiedades e as questões existenciais. Uma vez contemplados estes pontos, aumenta-se a probabilidade de personalizar a abordagem terapêutica e minimizar riscos ${ }^{1}$.

\section{EPIDEMIOLOGIA}

A aumentada expectativa de vida é uma realidade global, e o grupo dos indivíduos com 65 anos ou mais exibe uma curva de crescimento populacional rápida $^{2}$. Na última década, a proporção de pessoas acima de 65 anos aumentou, no oeste norte-americano, de $12,3 \%$ para $14,8 \%$, e na Suécia de $17,3 \%$ para $19,9 \%$. Na Ásia, esta mesma proporção aumentou de $19,8 \%$ para $26,3 \%$ no Japão, de 9,2\% para 13,1\% na Coreia do Sul, de 4,8\% para 5,6\% na Índia. Assim, 
o envelhecimento da população acompanha também a crescente incidência de DII no mundo - o que torna lógico o aumento no número de idosos diagnosticados. É fundamental que se possa definir dois paradigmas distintos: a doença no idoso diagnosticada anteriormente, na fase adulta, e a doença diagnosticada nos indivíduos com 60 anos ou mais, tema principal deste capítulo ${ }^{3}$.

No idoso, a retocolite ulcerativa (RCU) parece acometer mais os homens (56 a 62\%) do que as mulheres (38 a 44\%), enquanto a doença de Crohn (DC), ao contrário, ocorre mais em mulheres do que em homens ${ }^{1}$. A proporção de pacientes diagnosticados com DII aos 60 anos ou mais, demonstrada em uma série de estudos (Tabela 1), varia entre 3 e $21 \%$ para DC e de 7 a $29 \%$ para a RCU. A maioria (65\%) foi diagnosticada durante a $6^{\text {a }}$ década de vida, $25 \%$ na $7^{\mathrm{a}}$ e $10 \%$ na $8^{\text {a }}$ décadas ${ }^{4-12}$.

Tabela 1 Proporção de pacientes diagnosticados após os 60 anos entre todos os pacientes com doença inflamatória intestinal ${ }^{1,4-12}$

\begin{tabular}{|c|c|c|c|c|}
\hline Autor (ano) & País & $\mathrm{N}^{0}$ de pacientes & $\leq 60$ anos & $>60$ anos \\
\hline \multicolumn{5}{|l|}{ DC } \\
\hline Hwang et al. $(2017)^{5}$ & Coreia do Sul & 1.224 & 1.194 (97) & $30(3)$ \\
\hline Saygili et al. $(2017)^{8}$ & Turquia & 1.187 & $1.092(97)$ & $95(9)$ \\
\hline Nguyen et al. (2017) & Canadá & 8.985 & $8.260(92)$ & $725(8)^{a}$ \\
\hline Hou et al. (2016)7 & Estados Unidos & 724 & $632(87)$ & $92(13)^{a}$ \\
\hline Jeuring et al. (2016) & Holanda & 1.162 & $1.026(88)$ & $136(12)$ \\
\hline \multicolumn{5}{|l|}{$\mathrm{RCU}$} \\
\hline Song et al. (2018)9 & Coreia do Sul & 3.060 & $2.834(93)$ & $226(7)$ \\
\hline Nguyen et al. $(2017)^{6}$ & Canadá & 12.233 & $10.484(86)$ & $1.749(14)^{a}$ \\
\hline Lin et al. $(2016)^{10}$ & Taiwan & 536 & $459(86)$ & $77(14)$ \\
\hline Hou et al. $(2016)^{7}$ & Estados Unidos & 876 & $722(82)$ & $159(18)^{a}$ \\
\hline Jeuring et al. (2016) & Holanda & 1.661 & $1.288(78)$ & $373(22)$ \\
\hline Fries et al. $(2017)^{11}$ & Itália & 1.091 & $808(74)$ & $283(26)$ \\
\hline Shi et al. $(2016)^{12}$ & Hong Kong & 1.225 & $1.068(87)$ & $157(13)$ \\
\hline
\end{tabular}

Valores em porcentagem estão entre parênteses (\%).

Idade > 65 anos.

No idoso, a incidência de RCU é maior do que a de DC. Nos Estados Unidos, calcula-se a prevalência da DC em 240/100.000 e da RCU em $315 / 100.000$ habitantes com mais de 60 anos $^{13}$. Em um estudo húngaro, a incidência de RCU no idoso aumentou de 1,9/100.000 (anos de 1977-1981) para 10,8/100.000 (anos de 2002-2007), e a incidência da DC no idoso, vir- 
tualmente desconhecida até os anos de 1990, para 3,04/100.000 nos anos de 2002-2007 $7^{14}$. Em um estudo de impacto destas doenças na população asiática em 2010, a Índia foi o segundo país em impacto, após os Estados Unidos ${ }^{15}$. Assim, o paralelismo entre situações de melhor saneamento e a emergência de novos casos de DII é um dado crítico para um razoável entendimento da prevalência destas doenças no mundo em desenvolvimento e que pode auxiliar a desvendar pistas de prováveis associações do expossoma na etiologia da $\mathrm{DII}^{2}$.

\section{APRESENTAÇÃO CLÍNICA E LOCALIZAÇÃO DA DOENÇA}

A frequência da dor abdominal, da perda de peso e da febre na DII do idoso é mais baixa do que nos pacientes jovens, mas a abertura de um quadro de RCU do idoso, entretanto, pode ser mais grave. Em um trabalho holandês, a hospitalização ao diagnóstico ocorreu mais nos idosos com RCU do que na população mais jovem $(5,7 \% \text { versus } 2,9 \%)^{4}$. Nos casos de pacientes idosos com DC, há maior tendência ao sangramento retal, mas parece haver, realmente, menos dor abdominal, febre ou perda de peso na apresentação inicial. A DC no idoso habitualmente exibe envolvimento colônico e padrão inflamatório, com pouca ocorrência de fístulas ou estenoses, quando comparados aos fenótipos de pacientes mais jovens ${ }^{13}$. Em recente metanálise, a frequente localização colônica (até 52\% dos casos) foi seguida de ileal (32\%), íleo-colônica (26\%) e alta $(7 \%)^{1}$. Uma curiosidade é que o padrão e o comportamento da doença não costumam ser alterados entre os pacientes de 60 anos ou mais. Em relação à RCU, a apresentação clínica pode ser sutil, com poucos relatos de sangramento, diarreia ou dor, mas com abertura de quadro ou o primeiro episódio de reativação (o chamado flare, em inglês, ou recaída), sendo situações graves. Tipicamente, o paciente que permanece livre de cirurgias após a primeira recaída tem um prognóstico mais benigno, de longo prazo; outrossim, alguns casos, ao diagnóstico, necessitam de hospitalização ${ }^{13}$. A localização da RCU do idoso é mais frequente no cólon esquerdo, e o comportamento também costuma ser estável, conforme estudo de base populacional francês, que comenta não haver progressão proximal em $84 \%$ dos pacientes ${ }^{16}$.

Em uma recente metanálise, a RCU foi estudada quanto ao seu comportamento indolente, à luz da abordagem terapêutica: índices de cirurgia, uso de imunossupressores e terapia biológica. Os pacientes idosos foram mais propensos ao tratamento cirúrgico do que ao manejo com imunossupressores ou biológicos, e as altas taxas de cirurgia indicaram que, ou havia a preferência 
(pelos potenciais efeitos adversos com imunossupressores), ou a doença não era tão leve como se pensava. Em relação à DC, esta metanálise demonstrou que os pacientes idosos eram menos propensos a receber imunossupressores ou terapia biológica do que os pacientes mais jovens, mas a necessidade de cirurgia foi similar entre os dois grupos, desde o $1^{\circ}$ até o $5^{\circ}$ ano de acompanhamento. A progressão da doença permaneceu estável no grupo idoso ${ }^{1}$, e este dado é corroborado também pelas informações do registro francês EPIMAD $^{17}$, que exibe a estabilidade comportamental da DC nestes pacientes em $91 \%$ dos casos acompanhados no período médio de 6 anos ${ }^{1,17}$. De uma maneira geral, a DII do idoso representa alto risco de hospitalização, perfazendo $25 \%$ de todas as internações hospitalares por DII, o que demanda especial atenção médica no que diz respeito especialmente a eventos adversos de terapêuticas instituídas ou ao impacto da DII em comorbidades preexistentes. Os pacientes idosos tendem a ser menos tolerantes com o impacto causado pela DII quando comparados aos mais jovens, visto que situações como hipovolemia, desequilíbrio eletrolítico, anemia com recomendação de transfusão e desnutrição são indicações comuns de admissão hospitalar ${ }^{18}$. Este grupo é muito suscetível a infecções sérias - incluindo a colite pseudomembranosa -, é passível de longos períodos de permanência pós-operatória e tem alto risco de eventos tromboembólicos ${ }^{2,19-21}$.

\section{CONSIDERAÇÕES DIAGNÓSTICAS}

A investigação diagnóstica de DII no idoso deve assegurar, além da completa anamnese, que as potenciais interferências causadas pela própria condição do envelhecimento estejam sendo tratadas em ambiente o mais seguro possível. Quando este cenário não é contemplado, ocorrendo um atraso diagnóstico de média calculada em 7 anos, é deflagrada uma importante cascata de riscos acumulados. $\mathrm{O}$ atendimento médico, o exame físico e os exames complementares (laboratório e imagem, incluindo endoscopia) necessitam da adjuvância de informações específicas que corroborem com o diagnóstico diferencial e que levem em conta a polifarmácia, decorrente de comorbidades, usual neste grupo particular de pessoas. O diagnóstico diferencial da DII no idoso (Tabela 2) inclui a colite infecciosa, a colite isquêmica, a colite segmentar associada à diverticulite, a enteropatia pelo uso de anti-inflamatórios não hormonais (AINH), as doenças actínicas, a úlcera solitária de reto, a colite microscópica e as neoplasias. A doença colônica, o uso de corticosteroides e a imunossupressão são fatores de risco para a infecção por Clostridium difficile, cujas consequências podem ser extremamente graves. 
Tabela 2 Diagnóstico diferencial da doença inflamatória intestinal ${ }^{1}$

\begin{tabular}{|c|c|c|}
\hline Doença & Características clínicas & Observações \\
\hline $\begin{array}{l}\text { Colite } \\
\text { infecciosa }\end{array}$ & $\begin{array}{l}\text { Diarreia com sangue } \\
\text { Tenesmo } \\
\text { Histórico de febre } \\
\text { Início abrupto }\end{array}$ & $\begin{array}{l}\text { Possiveis pseudomembranas na } \\
\text { colite por Clostridium difficile } \\
\text { Cultura de fezes usualmente } \\
\text { diagnóstica } \\
\text { Resolução frequente com } \\
\text { adequada antibioticoterapia }\end{array}$ \\
\hline Colite actínica & $\begin{array}{l}\text { Diarreia com sangue } \\
\text { Dor abdominal e cólica } \\
\text { Proctite com tenesmo } \\
\text { Histórico de irradiação pélvica }\end{array}$ & $\begin{array}{l}\text { Telangiectasias observadas na } \\
\text { endoscopia e histologia }\end{array}$ \\
\hline $\begin{array}{l}\text { Enteropatia } \\
\text { por uso de } \\
\text { AINH }\end{array}$ & $\begin{array}{l}\text { Diarreia } \\
\text { Dor abdominal recorrente } \\
\text { Obstrução } \\
\text { Deficiência de ferro } \\
\text { Histórico de uso de AlNH }\end{array}$ & $\begin{array}{l}\text { Lesões isoladas } \\
\text { Qualquer porção do intestino } \\
\text { pode ser acometida } \\
\text { Estenoses anelares de intestino } \\
\text { delgado } \\
\text { Pode exacerbar DC ou RCU } \\
\text { preexistente }\end{array}$ \\
\hline $\begin{array}{l}\text { Colite } \\
\text { isquêmica }\end{array}$ & $\begin{array}{l}\text { Dor abdominal de início abrupto } \\
\text { Diarreia com sangue }\end{array}$ & $\begin{array}{l}\text { Distribuição segmentar } \\
\text { Ocorrência típica em sigmoide ou } \\
\text { cólon esquerdo } \\
\text { Reto preservado }\end{array}$ \\
\hline $\begin{array}{l}\text { Colite } \\
\text { segmentar } \\
\text { associada à } \\
\text { diverticulite }\end{array}$ & $\begin{array}{l}\text { Diarreia com sangue } \\
\text { Dor abdominal } \\
\text { História de comorbidades associadas, } \\
\text { como diabetes, hipertensão, doença } \\
\text { arterial coronariana }\end{array}$ & $\begin{array}{l}\text { Distribuição segmentar } \\
\text { peridiverticular } \\
\text { Reto preservado }\end{array}$ \\
\hline $\begin{array}{l}\text { Úlcera } \\
\text { solitária de } \\
\text { reto }\end{array}$ & $\begin{array}{l}\text { Sangramento retal } \\
\text { Tenesmo } \\
\text { Histórico de constipação crônica } \\
\text { Esforço evacuatório, evacuação digital }\end{array}$ & $\begin{array}{l}\text { Espessamento de mucosa } \\
\text { Distorção da arquitetura de criptas } \\
\text { Deposição de colágeno na lâmina } \\
\text { própria }\end{array}$ \\
\hline
\end{tabular}

AINH: anti-inflamatórios não hormonais; DC: doença de Crohn; DIl: doença inflamatória intestinal; RCU: retocolite ulcerativa.

A história familiar é um fator menos prevalente no diagnóstico da DII no idoso, bem como as manifestações extra-articulares, embora estas últimas possam apresentar-se em até $7 \%$ dos casos. Muita atenção deve ser dispensada às complicações tromboembólicas, possivelmente amparadas no status de hipercoagulabilidade, relativa imobilidade e desidratação associado às doenças. Não há evidência de que a terapia antiplaquetária desencadeie recaídas na DII, o que sugere ser prudente oferecer a aspirina aos pacientes com comorbidades cardiovas- 
culares associadas e indicação de uso, pois os dados a respeito desta proteção são consistentes ${ }^{2}$. Comorbidades frequentes aos idosos com DII seriam a hipertensão arterial, a doença cardiovascular, o diabetes melito, o acidente vascular cerebral (AVC), a doença pulmonar crônica obstrutiva (DPOC) e as malignidades. As comorbidades também podem afetar as escolhas terapêuticas, por exemplo: pacientes diabéticos e hipertensos não seriam candidatos ideais para a corticoterapia; a terapia inibidora do fator de necrose tumoral (anti-TNF) não é recomendada nos casos de insuficiência cardíaca congestiva (classes III/IV); e pacientes com história recente de malignidades podem não estar aptos à terapia com tiopurinas, visto que o risco de doenças mieloproliferativas é aumentado. Junto com a complexidade das escolhas, aparece a polifarmácia e seus riscos inerentes: os pacientes idosos podem estar utilizando, em média, 9 a 10 medicações, com o potencial de interações em um terço destas ${ }^{1}$. Além disso, o impacto com situações inesperadas, polievacuações, sintomas noturnos e incontinência leva ao uso de antidiarreicos, antiespasmódicos, antieméticos e analgésicos que são suscetíveis a efeitos anticolinérgicos. Analgésicos narcóticos, por exemplo, devem ser evitados, pelo alto potencial de alterar a motilidade digestiva no idoso e aumentar a probabilidade de infecções e índices de mortalidade ${ }^{22}$. Estes são dados que sinalizam a necessidade da atenção escrupulosa à história médica e às medicações concomitantes e que devem nortear a mais específica e otimizável terapêutica para a DII no idoso ${ }^{1}$.

\section{ABORDAGEM TERAPÊUTICA DA DOENÇA INFLAMATÓRIA INTESTINAL NO IDOSO}

O objetivo do tratamento da DII é modernamente compreendido como o alcance do estado de remissão profunda, com a ausência de sintomas clínicos e a cicatrização da mucosa, que agregam o benefício de diminuir taxas de hospitalizações, recaídas e cirurgias. Contudo, nem sempre o idoso está apto a tolerar a imunossupressão agressiva designada para esta finalidade. Assim, define-se o paciente ideal como aquele que apresenta mínima ou bem controlada comorbidade e que pode arcar com a estratégia terapêutica similar àquela aplicada em jovens, beneficiando-se, eventualmente, da intervenção precoce e protegendo-se dos graves desfechos atribuídos a longos períodos de atividade de doença ou ao uso de corticosteroides. Por outro lado, aos pacientes com pouco controle de comorbidades, impacto funcional e polifarmácia, reserva-se imperativo critério na seleção terapêutica, ponderação entre risco e benefício da imunossupressão e até mesmo a opção cirúrgica se houver contraindicações ou comportamento agressivo de doença, com estenoses e abscessos ${ }^{22}$.

Os aminossalicilatos, derivados do ácido 5 aminossalić́lico (5-ASA), são extensivamente utilizados na indução da remissão da RCU. Apesar de seu papel 
controverso na DC, são também prescritos para o idoso. No registro francês EPIMAD $^{1}$, os 5-ASA foram indicados para $75 \%$ dos pacientes com características fenotípicas similares, tanto de RCU quanto de DC. Entre os casos de DC, $68 \%$ receberam 5-ASA por 1 ano, $77 \%$ por 5 anos e $80 \%$ deles, por 10 anos. Entre $88 \%$ e $90 \%$ dos casos de DC colônica em terapia com 5-ASA, dois terços utilizaram a medicação por 10 anos, o que provoca e sugere algum papel, de fato, na DC do idoso ${ }^{23}$. Mais de $50 \%$ dos casos de RCU receberam 5-ASA em monoterapia e não necessitaram de cirurgia ${ }^{16}$. Sabe-se que a combinação oral e tópica é mais efetiva do que o uso somente oral e que regimes de dose única ao dia são preferíveis pela adesão. Além disso, apesar de apresentar bom perfil de segurança, foram descritos raros eventos de nefrotoxicidade e nefrite intersticial, e os sintomas mais comuns decorrentes do uso são náusea, vômito, dor abdominal, dor de cabeça e rash cutâneo. Potenciais interações seriam com a warfarina, aumentando a atividade anticoagulante da medicação, e com as tiopurinas, aumentando os níveis destas e o risco da mielossupressão ${ }^{1}$.

Os corticosteroides são os pilares da indução da remissão nos pacientes com RCU grave e com inadequada resposta aos 5-ASA, assim como na indução de remissão clínica nos casos de DC. Entretanto, os altos níveis de eficácia contrapõem-se aos riscos descritos, em até 6 meses de uso, de infecções sérias (pneumonia, infecção intestinal, enterocolite por C. difficile, são alguns exemplos) e as taxas de mortalidade associadas. Os eventos adversos relacionados à dose são reportados em $40 \%$ dos idosos que usam por longo prazo e a osteoporose, em $16 \%$ destes. Apesar destas informações, os idosos são frequentemente submetidos a estas terapias que, além do mais, necessitam de estratégias adequadas de desmame, o que muitas vezes também não ocorre ${ }^{1}$. Em um estudo de coorte norte-americano, com o objetivo de descrever o uso de esteroides na população, foi examinado um grupo representativo de 8.500 pacientes: os dados demonstraram que $20 \%$ dos pacientes que estavam recebendo terapia anti-TNF, haviam sido tratados por mais de 3 meses com corticosteroides, e que $10 \%$ usaram esteroide por mais de 6 meses. Ainda, $43 \%$ dos pacientes haviam recebido anti-TNF juntamente com corticosteroides em múltiplos cursos, por 3 meses. Em resumo, esse estudo indica a necessidade de planejamento na utilização de corticosteroides, mesmo em idosos idealmente selecionados ${ }^{24}$. Os corticosteroides são associados a outras morbidades, como alterações de estado mental, depressão, retenção hídrica, glaucoma, osteoporose, osteonecrose e fraturas. A má absorção e a deficiência de cálcio e de vitamina $\mathrm{D}$ no idoso sinaliza a necessidade de controle regular por meio de densitometria. Os corticosteroides podem reduzir a atividade de algumas medicações, como a fenitoína, o fenobarbital, a efedrina e a rifampicina, e podem afetar a coagulabilidade promovida pela warfarina, o que torna recomendável o controle laboratorial para particular monitoração ${ }^{1}$. 
Finalmente, as evidências publicadas sobre a indicação de corticosteroides na DII são unânimes em demonstrar o aumento de risco de eventos adversos sérios nos idosos quando comparados à população mais jovem².

Os imunossupressores podem ser empregados em idosos que demonstraram corticodependência ${ }^{2}$. Por outro lado, são medicações que necessitam de muita cautela, pois são associadas a alto risco de infecções oportunistas e também neoplasias. Apesar do metotrexato não ter sido especificamente estudado no idoso com DII, nenhum evento adverso foi reportado pelo uso nos idosos com artrite reumatoide ou psoríase, e também não foi associado à malignidades. O metotrexato tem sido atualmente utilizado na DC, mas não há consenso a respeito de sua aplicabilidade na $\mathrm{RCU}^{2,25}$. Em relação às tiopurinas, os eventos adversos nos idosos são comparáveis aos do grupo adulto de 18 a 40 anos de idade, sendo $46 \%$ e $32 \%(\mathrm{p}=0,001)$ respectivamente, e chama a atenção a mielotoxicidade e hepatotoxicidade ${ }^{13}$. A mielotoxicidade da tiopurina também pode ser deflagrada pela interação com o alopurinol, medicação razoavelmente utilizada pelo idoso para o manejo de distúrbios reumatológicos. Interessante é que o aluporinol também é considerado na otimização de dose da tiopurina, quando o paciente não atinge a concentração esperada do imunossupressor ${ }^{2}$. $\mathrm{O}$ uso destas substâncias no idoso exige cuidadoso monitoramento, considerando as interações medicamentosas e o aumento de risco de linfoma, câncer de pele não melanoma e infecções. A idade do idoso com DII é um fator de risco independente para eventos adversos decorrentes de medicações. A ciclosporina - outro imunossupressor muitas vezes indicado no manejo da DII do adulto - é muito evitada no paciente idoso com DII e, quando utilizada, deve ser monitorada criticamente, dado o seu potencial de nefrotoxicidade ${ }^{2}$.

O papel do anti-TNF na indução e na remissão da DII moderada a grave tem sido demonstrado há 20 anos, mas dados que forneçam suporte ao uso em idosos são controversos, limitados e escassos ${ }^{1,2}$. Algumas publicações exibem resultados similares entre os idosos e os mais jovens, mas outras chegam a sugerir menor eficácia ${ }^{26,27}$. Os dados de segurança dos anti-TNF nos idosos são consistentes em reportar alto risco de eventos adversos, como a publicação da Mayo Clinic na qual 3 de cada 4 mortes com o infliximabe ocorreram em idosos, apesar da incerta contribuição a estes desfechos, pelos longos cursos de doença, gravidade maior e múltiplas comorbidades que estes idosos expressavam em seus perfis ${ }^{27}$. Em relação à remissão livre de esteroides e às comparações pertinentes ao manejo dos pacientes não idosos, seja com monoterapia ou terapia biológica combinada com imunossupressor, não há robustas evidências no idoso e as amostras são pouco significativas; todavia, sabe-se que a imunossupressão combinada aumenta o risco de infecções oportunistas, incluindo a tuberculose. $\mathrm{O}$ uso de anti-TNF com imunossupressor também é associado ao 
aumento de risco para o desenvolvimento de linfoma não Hodgkin no adulto com DC, e este dado deve ser ponderado na tomada de decisão. O rastreamento prévio à terapia biológica deve ser realizado criteriosamente, e sugestões de rastreamento também para doença cardiovascular tem sido descritas em estudos recentes, cuja lógica é reforçada pela idade do idoso como fator de risco independente para uma série de comorbidades ${ }^{2}$.

As anti-integrinas são a novidade do arsenal terapêutico das DII. Em recente análise pos hoc, o vedolizumabe mostrou um perfil de segurança similar entre os grupos estudados, que incluíam pacientes acima de 55 anos, e que exibiram a menor incidência de infecções sérias ( 0,9 por 100 pacientes-ano) e eventos adversos que necessitassem de hospitalização (14,8 por 100 pacientes-ano). A especificidade intestinal e o perfil de segurança do vedolizumabe podem ser atrativos quando a monoterapia for desejada e estiver indicada ${ }^{28}$.

\section{CIRURGIA}

Aproximadamente um quarto das cirurgias intestinais ocorre em pacientes acima dos 55 anos, e a indicação mais frequente decorre da falha na terapia clínica ${ }^{1}$. A ocorrência de cirurgia entre as faixas etárias dos idosos e dos pacientes com diagnóstico mais jovem tem sido similar na RCU e na DC ${ }^{2}$. Entretanto, pela possível alteração esfinctérica no idoso, as opções de bolsa ileal e ileostomia têm sido ponderadas. A colectomia total com ileostomia permanente é o procedimento mais comum no idoso com RCU (77,3\%), enquanto a colectomia com bolsa ileal é a técnica de escolha entre os mais jovens. Esta discussão decorre de possíveis desfechos pós-operatórios, como é o caso dos distúrbios de função esfinctérica e incontinência fecal no paciente idoso ${ }^{1}$. Nos pacientes com DC, a necessidade de cirurgia parece ser mais baixa no grupo dos idosos; contudo, quando necessária para intervir nas complicações inerentes à doença, a abordagem técnica tem sido a mesma aplicada aos mais jovens: drenagem de abscessos, ressecção de estenoses ou estricturoplastia. Ainda que a morbidade e a mortalidade pós-operatória aumente com a idade, há muitas controvérsias entre os resultados descritos na população idosa com DII, bem como entre os dados de recorrência pós-operatória, e é conveniente recordar que, no idoso com DII, habitualmente o padrão da doença é mais estável ${ }^{1,2}$.

\section{CONSIDERAÇÕES FINAIS}

O entendimento ainda tímido sobre a imunopatogênese da DII, a própria definição de controle inflamatório e a ênfase na cicatrização da mucosa são itens que influenciam as decisões terapêuticas, com a precoce intervenção 
imunossupressora ou biológica em pacientes selecionados. No entanto, a questão é se estas tomadas de decisão cuidadosas realmente podem servir a todos. Um paciente idoso, com suas limitações e comorbidades controladas, pode ter desfechos mais razoáveis do que um paciente de qualquer idade que exponha fragilidades físicas ou riscos medicamentosos. Assim, há uma urgente necessidade de evidências sobre a biologia do envelhecimento e suas implicações na DII, pois correm em paralelo a crescente incidência e o aumento da expectativa de vida, cruzando-se em excelentes publicações e sendo uma temática ainda não atendida adequadamente pela academia.

\section{REFERÊNCIAS BIBLIOGRÁFICAS}

1. Kedia S, Limdi JK, Ahuja V. Management of inflammatory bowel disease in older persons: evolving paradigms. Intest Res. 2018;16(2):194-208.

2. Sturm A, Maaser C, Mendall M, Karagiannis D, Karatzas P, Ipenburg N, et al. European Crohn's and Colitis Organisation topical review on IBD in the elderly. J Crohns Colitis. 2017;11(3):263-73.

3. He W, Goodkind D, Kowal P. An aging world: 2015. Suitland: United States Census Bureau; 2016.

4. Jeuring SF, van den Heuvel TR, Zeegers MP, Hameeteman WH, Romberg-Camps MJ, Oostenbrug LE, et al. Epidemiology and long-term outcome of inflammatory bowel disease diagnosed at elderly age-an increasing distinct entity? Inflamm Bowel Dis. 2016;22(6):1425-34.

5. Hwang SW, Kim JH, Im JP, Ye BD, Koo HS, Huh KC, et al. Influence of age at diagnosis on the clinical characteristics of Crohn's disease in Korea: results from the CONNECT study. J Gastroenterol Hepatol. 2017;32(10):1716-22.

6. Nguyen GC, Bernstein CN, Benchimol EI. Risk of surgery and mortality in elderly-onset inflammatory bowel disease: a population-based cohort study. Inflamm Bowel Dis. 2017;23:218-23.

7. Hou JK, Feagins LA, Waljee AK. Characteristics and behavior of elderly-onset inflammatory bowel disease: a multi-center US study. Inflamm Bowel Dis. 2016;22:2200-5.

8. Saygili F, Saygili SM, Tenlik I, Yuksel M, Kilic ZM, Ozin YO, et al. Crohn's disease in the elderly: clinical presentation and manifestations from a tertiary referral center in Turkey. North Clin Istanb. 2017;3(3):183-6.

9. Song EM, Lee HS, Park SH, Kim GU, Seo M, Hwang SW, et al. Clinical characteristics and longterm prognosis of elderly-onset ulcerative colitis. J Gastroenterol Hepatol. 2018;33(1):172-9.

10. Lin WC, Tung CC, Lin HH, Lin CC, Chang CW, Yen HH, et al. Elderly adults with late-onset ulcerative colitis tend to have atypical, milder initial clinical presentations but higher surgical rates and mortality: a Taiwan Society of Inflammatory Bowel Disease study. J Am Geriatr Soc. 2016;64(10):e95-e97.

11. Fries W, Viola A, Manetti N, Frankovic I, Pugliese D, Monterubbianesi R, et al. Disease patterns in late-onset ulcerative colitis: results from the IG-IBD “AGED study”. Dig Liver Dis. 2017;49(1):1723.

12. Shi HY, Chan FK, Leung WK, Li MK, Leung CM, Sze SF, et al. Natural history of elderly-onset ulcerative colitis: results from a territory-wide inflammatory bowel disease registry. J Crohns Colitis. 2016;10(2):176-85.

13. Afzali A, Katz S. Inflammatory bowel disease in the baby to baby boomer: pediatric and elderly onset of IBD. Curr Treat Options Gastroenterol. 2018. [Epub ahead of print]

14. Lakatos PL, David G, Pandur T, Erdelyi Z, Mester G, Balogh M, et al. IBD in the elderly population: results from a population-based study in Western Hungary, 1977-2008. J Crohns Colitis. 2011;5(1):5-13. 
15. Singh P, Ananthakrishnan A, Ahuja V. Pivot to Asia: inflammatory bowel disease burden. Intest Res. 2017;15:138-41.

16. Charpentier C, Salleron J, Savoye G, Fumery M, Merle V, Laberenne JE, et al. Natural history of elderly-onset inflammatory bowel disease: a population-based cohort study. Gut. 2014;63(3):423-32.

17. Duricova D, Sarter H, Savoye G, Leroyer A, Pariente B, Armengol-Debeir L, et al. Impact of extra-intestinal manifestations at diagnosis on disease outcome in pediatric - and elderly-onset Crohn's disease: a French population-based study. Inflamm Bowel Dis. 2018. [Epub ahead of print]

18. Ananthakrishnan AN, McGinley EL, Binion DG. Inflammatory bowel disease in the elderly is associated with worse outcomes: a national study of hospitalizations. Inflamm. Bowel Dis. 2009;15:1829.

19. Ananthakrishnan AN, McGinley EL, Binion DG. Excess hospitalisation burden associated with Clostridium difficile in patients with inflammatory bowel disease. Gut. 2008;57:205-10.

20. Lichtenstein GR, Feagan BG, Cohen RD, Salzberg BA, Diamond RH, Price S, et al. Serious infection and mortality in patients with Crohn's disease: more than 5 years of follow-up in the TREAT registry. Am J Gastroenterol. 2012;107(9):1409-22.

21. Nguyen GC, Sam J. Rising prevalence of venous thromboembolism and its impact on mortality among hospitalized inflammatory bowel disease patients. Am J Gastroenterol. 2008;103:2272-80.

22. Ha CY, Katz S. Clinical implications of ageing for the management of IBD. Nat Rev Gastroenterol Hepatol. 2014;11:128-38.

23. Limdi JK. Aminosalicylates and elderly-onset colonic Crohn's disease-more than meets the eye? J Crohns Colitis. 2017;11:1022.

24. Johnson SL, Bartels CM, Palta M, Thorpe CT, Weiss JM, Smith MA. Biological and steroid use in relationship to quality measures in older patients with inflammatory bowel disease: a US Medicare cohort study. BMJ Open. 2015;5:e008597.

25. Magro F, Gionchetti P, Eliakim R, Ardizzone S, Armuzzi A, Barreiro-de Acosta M, et al. Third European evidence-based consensus on diagnosis and management of ulcerative colitis. Part 1 : definitions, diagnosis, extra-intestinal manifestations, pregnancy, cancer surveillance, surgery, and ileo-anal pouch disorders. J Crohns Colitis. 2017;11:649-70.

26. Desai A, Zator ZA, de Silva P, Nguyen DD, Korzenik J, Yajnik V, et al. Older age is associated with higher rate of discontinuation of anti-TNF therapy in patients with inflammatory bowel disease. Inflamm Bowel Dis. 2013;19(2):309-15.

27. Colombel JF, Loftus EV Jr., Tremaine WJ, Egan LJ, Harmsen WS, Schleck CD, et al. The safety profile of infliximab in patients with Crohn's disease: the Mayo clinic experience in 500 patients. Gastroenterology. 2004;126(1):19-31.

28. Yajnik V, Khan N, Dubinsky M, Axler J, James A, Abhyankar B, et al. Efficacy and safety of vedolizumab in ulcerative colitis and Crohn's disease patients stratified by age. Adv Ther. 2017;34(2):542-59. 